\title{
A l'école : des filles et des garçons mais pour quelle égalité ?
}

\section{Céline Delcroix}

\section{OpenEdition}

\section{Journals}

Édition électronique

URL : http://journals.openedition.org/trema/3568

DOI : $10.4000 /$ trema.3568

ISSN : 2107-0997

Éditeur

Faculté d'Éducation de l'université de Montpellier

Édition imprimée

Date de publication : 1 décembre 2016

Pagination : 1-3

ISBN : 979-10-96627-02-8

ISSN : 1167-315X

Référence électronique

Céline Delcroix, «A l'école : des filles et des garçons mais pour quelle égalité ? », Tréma [En ligne],

46 | 2016, mis en ligne le 01 avril 2017, consulté le 20 avril 2019. URL : http://journals.openedition.org/ trema/3568 ; DOI : 10.4000/trema.3568

Ce document a été généré automatiquement le 20 avril 2019.

Trema 


\title{
A l'école : des filles et des garçons mais pour quelle égalité?
}

\author{
Céline Delcroix
}

1 Depuis plusieurs années, la recherche internationale comme française ont largement posé des constats d'acquis, d'interactions, d'orientations, et de violences différenciées au sein de l'espace scolaire. Or, ces postulats qui engagent une réflexion sur les problématiques de l'égalité et des rapports sociaux au sein de l'École trouvent peu ou pas d'opérationnalisation pour les personnels enseignants. D'autant que la virulence des débats publics autour de divers modes de parentalité et de sexualités en 2014 et, parallèlement, leurs rejaillissements sur les pratiques enseignantes en termes d'égalité ont réactivé des questionnements sur la place des filles et des garçons à l'École.

2 C'est pourquoi, pour ce numéro, notre volonté est d'interroger les pratiques enseignantes, les discours, les politiques scolaires, et les formations qui prennent en compte les questions d'égalité dans leurs réponses multiples et variées face aux fortes tensions qui émergent au sein de l'espace scolaire.

3 Notre propos est de présenter avec une perspective genrée des contributions à la recherche qui ont toutes comme objectif de permettre à l'école de remplir sa mission d'égalité d'éducation et d'inclusion. Il s'agit de mettre au jour tout ce qui, dans le système scolaire, est invisibilisé en termes de savoirs et de personnes et qui cependant contribue activement à la reproduction des inégalités sociales et sexuées. Nous souhaitons donc défendre la valeur heuristique de réflexions autour des rapports sociaux.

4 Muriel Salle, Université Claude Bernard Lyon ESPE de Lyon, Laboratoire de Recherche Historique en Rhône Alpes (LARHRA) propose une réflexion sur l'ensemble des textes produits, conventions bilatérales puis interministérielles, par le ministère de l'Éducation nationale depuis trente ans sur l'égalité des Filles et des Garçons. L'auteure analyse les transformations lexicales en ce qu'elles révèlent les positions institutionnelles sur les problématiques d'égalité. Quelle place pour la mixité des sexes ? pour l'égalité ? pour la culture de l'égalité? 
5 Gaël Pasquier, ESPE de Créteil - Université Paris Est Créteil (UPEC), Observatoire Universitaire International Éducation et Prévention (OUIEP), Laboratoire Interdisciplinaire de Recherche sur les Transformations des pratiques Éducatives et des pratiques Sociales (LIRTES) révèle la position paradoxale d'enseignant-e-s du primaire qui prennent en compte la question de l'égalité des sexes par son analyse d'entretiens. En effet, ces enseignant-e-s font figure d'enseignant-e-s militant-e-s ou engagé-e-s au sein des équipes pédagogiques, et ce alors qu'ils/elles mobilisent dans leur pratique une préconisation des politiques éducatives.

6 Sigolène Couchot-Schiex, ESPE de Créteil - Université Paris Est Créteil (UPEC), Observatoire Universitaire International Éducation et Prévention (OUIEP), Laboratoire Interdisciplinaire de Recherche sur les Transformations des pratiques Éducatives et des pratiques Sociales (LIRTES) propose un parcours d'intégration de cette question vive que sont l'égalité des sexes et le genre dans les compétences professionnelles des enseignante-s en formation. D'autant que faire adopter une " démarche adaptative " selon les situations d'enseignement n'est pas sans effets sur la transformation professionnelle et personnelle des personnes concernées.

7 Loïc Szerdahelyi, Université Lyon 1, Chercheur associé au Laboratoire sur les Vulnérabilités et l'Innovation dans le Sport (L-VIS) EA 7428 démontre que l'intégration des femmes au niveau secondaire dans une discipline dite masculine, l'EPS, apporte un changement et modèle les attendus professionnels. En effet, l'auteur en saisissant le concept vichyste des "femmes d'action » propose une lecture actualisée de rapports d'inspection selon un ordre normatif : le genre et ses catégorisations du féminin et du masculin et ce dans un contexte de mixité des corps et des espaces.

8 Caroline Dayer, Université de Genève, Équipe Approche Compréhensive des Représentations et de l'Action (ACRA) analyse le processus de socialisation en négatif dans l'espace scolaire suisse. L'auteure souligne dans quelle mesure les violences homophobes à l'École élaborent des situations de vulnérabilité pour les élèves touché-e-s ce qui n'est pas sans effet sur leurs apprentissages. Elle montre comment l'hétérosexisme mobilise une socialisation en creux, par défaut pour les élèves qui connaissent des violences sexistes et homophobes en milieu scolaire.

9 Isabelle Collet, Université de Genève, Groupe relations interculturelles et formation des enseignants - genre et éducation (Grifege) rappelle que le constat des choix d'orientation différenciés pour les filles et les garçons est réalisé depuis 1984 et ce notamment pour les filières scientifiques. L'auteure analyse, tout d'abord, les mécanismes entravant les filles à choisir des études scientifiques puis par son enquête de terrain sur le curriculum réel, montre dans quelle mesure et sous quelles formes les Sciences sont introduites à l'école primaire en Suisse.

10 Cendrine Marro, Université Paris Ouest Nanterre La Défense, Laboratoire d'études de genre et de sexualité (LEGS) UMR 8238, Gaël Pasquier, ESPE de Créteil, Université Paris Est Créteil (UPEC), Observatoire Universitaire International Éducation et Prévention (OUIEP), Laboratoire Interdisciplinaire de Recherche sur les Transformations des pratiques Éducatives et des pratiques Sociales (LIRTES) et Laurence Breton, Conseillère pédagogique chargée de la Maîtrise de la langue et de l'Enseignement moral et civique proposent un premier partage de leur recherche action sur l'éducation à l'égalité des sexes et la littérature de jeunesse. Les auteurs partent du constat que sensibiliser aux inégalités n'est pas seulement énoncer le principe d'égalité. Ils développent une 
démarche de lecture critique sur des textes littéraires qui interroge l'égalité des sexes versus les inégalités par le ressenti émotionnel. En effet, éveiller les élèves à l'empathie par la compréhension d'ouvrages de littérature de jeunesse est un ressort efficace dans la lutte contre les préjugés (comme l'ont démontré d'autres disciplines).

11 Céline Delcroix, ESPE de Créteil SPE de Créteil, Université Paris Est Créteil (UPEC), Observatoire Universitaire International Éducation et Prévention (OUIEP), Laboratoire Interdisciplinaire de Recherche sur les Transformations des pratiques Éducatives et des pratiques Sociales (LIRTES). Pour ma part, selon une approche socio-historique, j'analyse la formation du corps des enseignant-e-s du primaire selon une logique ségrégative. Il s'agit en effet de souligner comment un maintien des séparations des sexes et des classes a longtemps opéré dans l'enseignement primaire. Car, à sa masculinisation première a répondu une féminisation massive dès le XIXe siècle. Et, aujourd'hui, à l'école primaire, les enseignants sont majoritairement des femmes issues des classes favorisées quand autrefois ils étaient des hommes issus du peuple.

\section{AUTEUR}

\section{CÉLINE DELCROIX}

ESPE de Créteil - Université Paris Est Créteil, Observatoire Universitaire International Éducation et Prévention (OUIEP), Laboratoire Interdisciplinaire de Recherche sur les Transformations des pratiques Éducatives et des pratiques Sociales (LIRTES) 